\title{
Evaluación de Tecnologías Sanitarias Cirugías para el tratamiento de la obesidad mórbida
}

\section{Contexto Clínico}

La obesidad es una condición cada vez más prevalente en todo e mundo. Se la denomina obesidad mórbida cuando el índice de masa corporal (IMC) es mayor a 40, o cuando este supera los 35 pero el paciente presenta comorbilidades.

En Argentina ${ }^{1,2}$, la prevalencia de obesidad es de 20 a 30\%. Ser obeso se asocia a un incremento en la mortalidad, así como un aumento de la morbilidad asociada a osteoartritis, lumbalgia, diabetes, hipertensión, hernia hiatal, enfermedad vesicular, disnea e hipercolesterolemia. Además, la obesidad constituye un factor de riesgo independiente de enfermedad coronaria.

En general, la obesidad es tratada principalmente con dietas, ejercicio físico y cambios en el estilo de vida. También se pueden utilizar drogas que reducen la absorción de grasas, como el orlistat o que alteran los niveles de noradrenalina y serotonina, como la sibutramina o la fluoxetina, brindando sensación de saciedad. Sin embargo, en ocasiones, estos tratamientos no son efectivos, por lo que se ha propuesto la realización de tratamientos quirúrgicos cuyo objetivo es la reducción y el mantenimiento posterior del peso mediante una restricción en la ingesta y/o absorción de alimentos.

\section{Descripción de la tecnología}

Diversas técnicas quirúrgicas han sido propuestas para el tratamiento de la obesidad mórbida. Entre ellas puede mencionarse principalmente al by-pass gástrico (variaciones de la cirugía de Roux en Y o "Y de Roux") la colocación de una banda en el estómago (ajustable o no ajustable) la gastroplastia vertical u horizontal y la derivación biliopancreática con "switch" duodenal. Cabe aclarar que algunos de estos procedimientos pueden realizarse por vía laparoscópica o por vía convencional.

\section{Objetivo}

Evaluar la eficacia y seguridad de diversas técnicas quirúrgicas para el tratamiento de la obesidad mórbida.

\section{Métodos}

Se realizó una búsqueda en las principales bases de datos bibliográficas (MEDLINE, Cochrane, DARE, NHS EED) en buscadores genéricos de Internet, en sitios de agencias de evaluación de tecnologías sanitarias y financiadores de salud utilizando las siguientes palabras claves: "morbid obesity" y "surgery". También se buscaron evaluaciones de tecnologías sanitarias y evaluaciones económicas, guías de práctica clínica y políticas de cobertura de otros sistemas de salud. Se buscaron datos de fuentes locales sobre la utilización de recursos sanitarios, costos y cobertura en Argentina.

\section{Resultados}

Dado que la colaboración Cochrane llevó a cabo una revisión sistemática sobre este tema en $2001^{3}$, se realizó una búsqueda desde esa fecha en adelante, priorizando las revisiones sistemáticas. De otras seis revisiones sistemáticas halladas, se comenta con mayor detalle la realizada por Buchwald y col. en 2004.

La Colaboración Cochrane realizó una revisión sistemática que incluyó 18 estudios, de los cuales 16 ensayos clínicos controlados aleatorizados (ECCA) comparan las diferentes técnicas quirúrgicas entre sí, mientras que un ECCA y un estudio no aleatorizado compararon los procedimientos quirúrgicos con tratamientos médicos. Los individuos analizados eran en su mayoría de sexo femenino, de 33 a 47 años y con un peso entre 115 y 175 kilogramos.

Buchwald y col. ${ }^{4}$ publicaron una revisión sistemática que analizó 136 estudios (de los cuales sólo cinco era ECCA, en su gran mayoría series de casos). La población en estudio era en más de un $70 \%$ de sexo femenino, con una edad promedio de 39 años y un IMC basal promedio de 46,8 (rango entre 32 y 69 ).

\section{Pérdida de peso}

La revisión de Cochrane muestra que con la gastroplastia horizontal (un ECCA) se obtiene una reducción del peso superior a la de una dieta de bajas calorías (entre 23 y $28 \mathrm{~kg}$ en dos años).

Los tratamiento quirúrgicos (excepto con derivación biliopancreática para el cual la evidencia es escasa) son más efectivos que los no quirúrgicos, con una reducción de 25 a $44 \mathrm{~kg}$ en un plazo de uno y/o dos años, y con una pérdida de peso sostenida de $20 \mathrm{~kg}$ a los ocho años.

En la publicación de Buchwald y $\mathrm{col}^{4}$., el promedio de pérdida del exceso de peso fue de $47,5 \%$ para el bandeo gástrico, $61,6 \%$ para el "bypass" gástrico, 68,2\% para la gastroplastia y $70,1 \%$ para la derivación biliopancreática con "switch" duodenal. La reducción media del IMC fue de 14,2. En todos los casos las reducciones fueron estadísticamente significativas.

\section{Mortalidad operatoria}

La mortalidad perioperatoria se encuentra entre $0 \%$ y $1,5 \%$, sin diferencias significativas entre las diferentes técnicas.

Buchwald y col publicaron una mortalidad operatoria temprana (los primeros 30 días) del $0,1 \%$ para los procedimientos puramente restrictivos, $0,5 \%$ para los "bypass" gástricos y $1,1 \%$ para las derivaciones biliopancreáticas con "switch" duodenal. Las complicaciones más frecuentes fueron: abscesos subfrénicos (7\%) atelectasia o neumonía (4\%) síntomas pulmonares $(6,2 \%)$ e infecciones de la herida (4\%).

Comorbilidades

Se observaron beneficios como normalización de la glucemia, reducción de los niveles de tensión arterial y mejoría en los perfiles lipídicos. Sin embargo, no hay datos concluyentes publicados sobre estos datos a largo plazo ni en referencia a la mortalidad.

\section{Comparación entre técnicas}

- Bandeo gástrico vs. gastroplastia vertical usando una banda: la colaboración Cochrane reporta que quienes realizaron bandeo gástrico perdieron menos peso en estudios con seguimiento entre uno y tres ${ }^{5}$, pero con resultados algo mejores a los cinco años (43 vs. $35 \mathrm{~kg}$ ). En cuanto a las complicaciones, los resultados son equívocos según las diversas publicaciones, sin diferencias en mortalidad.

Bandeo gástrico vs. "by pass" gástrico: no se encontraron resultados concluyentes sobre mayor beneficio de una técnica sobre la otra, ni sobre alguna diferencia en cuanto al riesgo de complicaciones. Algunos trabajos muestran una pequeña ventaja a corto plazo del "by-pass" gástrico.

Gastroplastia horizontal vs. "by pass" gástrico: se ha reportado una mayor pérdida de peso a 12 meses en el "by-pass" gástrico que en la gastroplastia horizontal $(35-42 \%$ vs. $16-29 \%, p<0,05) \sin$ diferencias significativas en la mortalidad perioperatoria.

\section{Vía de abordaje quirúrgica}

Las diferentes técnicas abordadas por laparoscopía han mostrado ser relativamente seguras, con menor estadía hospitalaria y menores tasas de readmisión y complicaciones . Sin embargo, aún no se conoce la eficacia de las mismas a largo plazo.

\section{Implicancias económicas}

Un estudio de costo-efectividad 6 realizado por la agencia de evaluaciones tecnológicas de salud de Southampton (SHTAC) con un horizonte a 20 años concluyó que, en comparación con las terapéuticas tradicionales, los tratamientos quirúrgicos, tienen un mayor beneficio clínico con un costo adicional.

\section{Conclusiones}

Si bien la evidencia disponible sugiere mayores tasas de pérdida de peso con la cirugía que con tratamientos no quirúrgicos, no se han encontrado datos concluyentes respecto de las comorbilidades y la calidad de vida en el largo plazo. Debe tenerse en cuenta que 
la mayor parte de los estudios fueron realizados en individuos con un IMC mayor a 35 ó 40 y que no habían podido disminuir su peso con tratamiento médico convencional. De todas las técnicas quirúrgicas, la más aceptada y difundida es el "by-pass" gástrico.

Las diferentes técnicas abordadas por laparoscopía han mostrado ser relativamente seguras, con menor estadía hospitalaria y menores tasas de readmisión y complicaciones. Sin embargo, aún no se conoce la eficacia de las mismas a largo plazo.

Se trata de un tratamiento de gran complejidad que además de implicar un procedimiento quirúrgico mayor, afecta al paciente en forma importante en su esfera física, psicológica y social. Por estos motivos hay cierto consenso en indicarlo sólo cuando se cumplen los siguientes criterios:

- IMC mayor a 40 con más de cinco años de evolución.

- IMC mayor a 35 en individuos con comorbilidades importantes que mejorarían al mejorar la obesidad.

- Al menos un año documentado de intentos de descenso de peso que hayan incluido dieta, ejercicio y tratamientos farmacológicos.

- Evaluación psicológica de la aptitud del paciente para someterse a un tratamiento de estas características.

- Edad mayor de 18 años

Modificado de: Pichon Riviere A, Augustovski F, Ferrante D, García Martí S, Glujovsky D, López Analía, Regueiro A. Instituto de Efectividad Clínica y Sanitaria (www.iecs.org.ar). Utilidad de los tratamientos quirúrgicos en la obesidad. Documentos de Evaluación de Tecnologías Sanitarias, Informe de Respuesta Rápida № 44. Buenos Aires, Argentina. Abril de 2005. Fecha de Última Actualización 04/2004.

\section{Referencias}

1. De Sereday, M.S., et al., Prevalence of diabetes, obesity, hypertension and hyperlipidemia in the central area of Argentina. Diabetes Metab, 2004. 30(4): p. 335-9.

2. Carbajal, H.A., et al., [Associated variables to hypertension in a region of Argentina]. Medicina (Bs. Aires), 2001. 61(6): p. 801-9.

3. Colquitt, J., et al., Surgery for morbid obesity. Cochrane Database Syst Rev, 2003(2): p. CD003641.

4. Buchwald, $H$., et al., Bariatric surgery: a systematic review and meta-analysis. Jama, 2004. 292(14): p. 1724-37.

5. Chapman, A.E., et al., Laparoscopic adjustable gastric banding in the treatment of obesity: a systematic literature review. Surgery, 2004. 135(3): p. 326-51. 6. Clegg, A., et al., Clinical and cost effectiveness of surgery for morbid obesity: a systematic review and economic evaluation. Int J Obes Relat Metab Disord, 2003. 27(10): p. 1167-77.

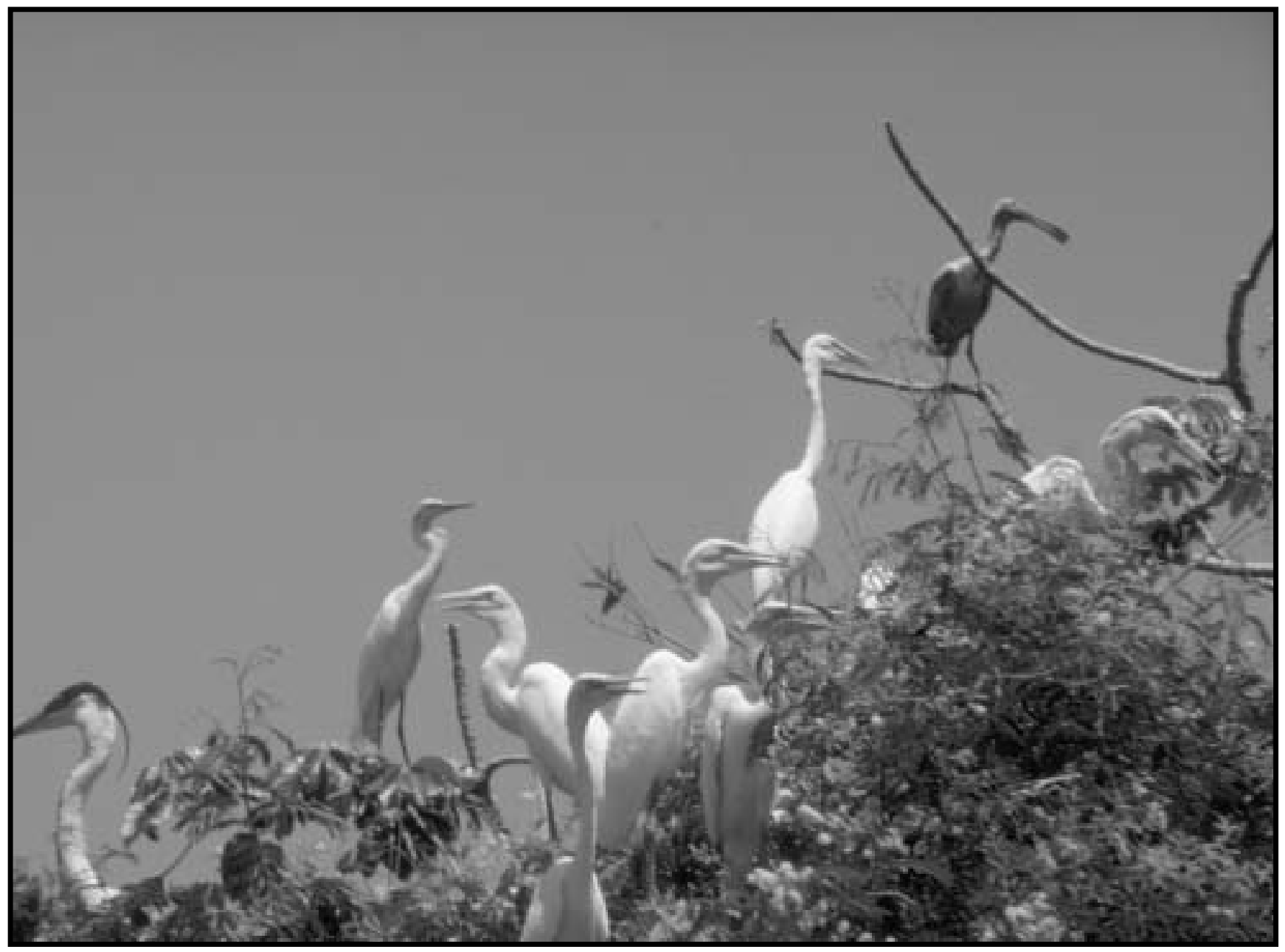

Gentileza Secretaria de Turismo de La Nación 